At least several types of human haematological malignancies can now be seen as 'stem-cell diseases'. The best-studied in this context is acute myeloid leukaemia (AML). It has been shown that these diseases are driven by a pool of 'leukaemia stem cells (LSC)', which remain in the quiescent state, have the capacity to survive and self-renew, and are responsible for the recurrence of cancer after classical chemotherapy. It has been understood that LSC must be eliminated in order to cure patients suffering from haematological cancers. Recent advances in LSC research have allowed for description of LSC phenotype and identification of potential targets for anti-LSC therapies. This concise review summarises the current view on LSC biology and targeted approaches against LSC.

Key words: leukaemia stem cell, selfrenewal, cancer recurrence, chemotherapy resistance, targeted therapies.

Contemp Oncol (Pozn) 2015; 19 (1A):

A1-A6

DOI: $10.5114 /$ wo.2014.47127

\section{Cancer stem cells in haematological malignancies}

\author{
Radoslaw Zagozdzon, Jakub Golab
}

Department of Immunology, Centre of Biostructure Research, Medical University of Warsaw, Poland

\section{Introduction}

Since the first application of nitrogen mustard derivatives to treat haematological malignancies in the 1940s, for decades the general idea of anticancer therapies was to eradicate as high a percentage of tumour cells as possible by applying the maximum tolerated dose of a toxic agent. Such agents were mainly designed to eliminate rapidly dividing cells as they were thought to be the most prominent feature of cancer in general. One of the immediate observations was, however, that even a vast reduction of cancer content in the patient's organism can be transient and subsequent recurrence can occur. This, along with parallel observations of robust heterogeneity among cancer cells, led to the idea of the existence of a distinct pool of 'cancer-initiating cells', which do not divide rapidly and thus are relatively resistant to classical chemotherapeutics, and which possess the ability to reconstitute the cancer bulk after therapy. That idea has deeply changed the understanding of therapeutic needs in cancer. This review briefly summarises the current state-of-the-art understanding of the role of cancer-initiating cells in haematological cancers. Unless otherwise stated, most of the information reviewed in this work relates to acute myeloid leukaemia (AML), as the best-studied disease in this context.

\section{Stem cell paradigm in haematopoietic malignancies}

Intratumoural heterogeneity spotted in most of the malignant cancers has puzzled researchers for decades. Two main models have been proposed to explain this phenomenon: the 'stochastic model' and the 'stem cell model'.

\section{Stochastic model}

The stochastic model is historically older [1]. Most of the cancer cells would have the same potential to self-renew and divide, and the choice between self-renewal and differentiation would be more or less random unless one of them is induced by external factors. Under this model, practically each cancer cell could reproduce the whole cancer. Also under this model, cancer existence would be mostly dependent on symmetric divisions of cancer cells, each of them giving rise to identical progeny. Cancer growth would then be highly disorganised, and only a small, random number of cancer cells would be arrested in the GO phase of the cell cycle, and these cells would be responsible for the regrowth of cancer after chemotherapy.

\section{Stem cell model}

Opposing the stochastic model, the stem cell theory was proposed in the 1940s [2] and experimentally validated later on [3]. This concept assumes that cancers, especially haematological malignancies, resemble the hierarchical organisation of healthy haematopoiesis. In this model, a distinct set of stem cells would undergo a slow, asymmetrical division to produce an identical stem cell (self-renewal) and at the same time to produce a more differentiated progenitor cell. The progenitors would then undergo rapid 
divisions to generate most of the cancer bulk within the given patient.

Multiple laboratories devoted their efforts to identify, describe, and target cancer stem cells. A great deal of progress has recently been made, especially in the field of haematological malignancies. However, as usual for cancer-related studies, the image generated by this research appears to be far more complicated than a simplistic assumption of one stem cell pool responsible for cancer regeneration. The landscape of 'stemness' in haematological malignant disorders is characterised by considerable complexity and dynamics, and is still a matter of intensive debate.

Given the fact that cancer can be mostly seen as a disease of the genes, the stem sell model raises the question of whether a sequence of gene alterations occurs in a healthy haematopoietic stem cell or, alternatively, a highly committed progenitor de-differentiates into stemlike tumour-initiating cell. Currently, while the occurrence of the latter situation cannot be excluded in some cases, more evidence has been gathered in support to the first option. That especially holds true for AML and myelodysplastic syndromes (MDS). Under this model, the process of leukaemia development (leukaemogenesis) would rely on a series of stepwise mutations within the primarily immature, multipotent, and quiescent haematopoietic stem or early progenitor cell (HSC). At some point, these transforming events accumulate to give rise to a 'leukaemia-cell of origin' (LCO), which is still considered to be a 'preleukaemic stem cell' (pre-LSC) [4]. If such cells are xenotransplanted to an immunocompromised host, they would produce leukaemia with variable penetration and considerably long latency. These cells need further genetic and/or epigenetic events to develop into a 'leukaemic stem cell' (LSC), capable of producing fully penetrant leukaemic growth with short latency in the xenotransplantation models.

It is important to understand the functional alterations harboured by LSC in comparison with the healthy HSC. The most obvious of these are reduced differentiation, decreased apoptosis, and deep metabolic changes. Also crucial is the alteration in the self-renewal and proliferative capabilities; while normal HSC divide mostly in an asymmetric manner, in LSC the chance for symmetric division producing two identical stem cells is greatly increased, as shown by mathematical modelling [5].

\section{Heterogeneity of leukaemia stem cells}

A great deal of evidence has been gathered in support of the hierarchical organisation of the leukaemic growth, resembling to some extent the natural haematopoiesis process. What must be remembered, however, is that LSC are characterised by dramatically increased genetic instability. This translates into an increased chance of random events within their genome and, during subsequent symmetrical divisions, would eventually translate into generation of a number of LSC subclones within a given cancer. All surviving LSC subclones would retain their self-renewal and leukaemia-initiating capabilities, but may gain new oncogenic drivers and differentially respond to the therapeutic approaches. If true, this model has profound impli- cations for targeted therapies in leukaemia and explains why 'narrow-spectrum' targeted therapies have a considerably high chance of failure in the long term, even if they are designed to disturb functioning of LSC. It would also explain difficulties in unequivocal description of the molecular phenotype of the 'universal' LSC.

\section{Methods of identification of haematopoietic cancer stem cells}

\section{Xenotransplant models}

Identification of the LSC via xenotransplant-based models relies on the determination of whether a given cell type stably reproduces all populations of the original malignancy in the recipient animal. The first evidence, based on the xenotransplantation models, for the functioning of LSC in human haematopoietic malignancies has been provided in the 1990s [6, 7]. The main limitation for this assay is the fact that it depends on the ability of studied cells to home to the mouse bone marrow, which does not necessarily have to be a feature of a human stem cell. The other issue is related to differences between humans and mice regarding the microenvironmental conditions within the stem cell niches in bone marrow. Nevertheless, the limiting dilution analysis (LDA) via transplantation to severe combined immunodeficient (SCID), nonobese diabetic (NOD)/ SCID, or, recently, NOD/SCID/IL2rynull (NSG) mice has become one of the standard methods for functional identification of LSC. The LSC frequency identified by these techniques varies and is estimated from approximately 1 in $1.6 \times 10^{3}$ to 1 in $1.1 \times 10^{6}$ cells, and was shown to be usually highest in the $\mathrm{CD} 34^{+} \mathrm{CD} 38^{-}$fraction [8]. Conspicuously, higher frequencies of stem cells in acute myeloid leukaemia were shown to correlate with robust minimal residual disease and poorer survival of patients [9].

\section{Cell surface markers}

While molecular phenotype of LSC relates to multiple features in various cellular compartments, in the era of flow cytometry-based cell sorting the utilization of cell surface markers provides a high-level convenience for isolation of LSC. Initial studies were focused on the CD34+CD38- Lin ${ }^{-}$ population of leukemic cells [7]. Further on, LSC phenotype was shown far more complex and numerous other markers were proposed useful in LSC phenotyping. These include CD71, CD90, CD117, CD123, HLA-DR [10], or CD33 [11] for AML, CD26 for chronic myeloid leukaemia (CML) [12], or CD133 for acute lymphoblastic leukaemia (ALL) [13].

From a therapeutic point of view, it is important to identify the cell surface markers that can distinguish between normal HSC and LSC. Normal HSC are usually of the following phenotype: CD34+/CD38-/CD13-/CD33+/CD90+/ CD123-lo/CD117+/CD71+ [14]. Aberrant surface phenotype of AML stem cells usually lacks the expression of CD90, CD117, and HLA-DR but is robust for CD123, CD96, CD44, C-type lectin-like molecule-1 (CLL-1) [15], or CD47 [16]. There is some debate on the expression of CD33 molecule on AML LSC [11] and its importance for anti-AML therapies. Interestingly, unlike regular HSC, the LSC can sometimes 
be devoid of CD34 expression [17]. New markers are also being proposed based on proteomic analyses [18].

\section{In vitro functional assays}

As the xenotransplantation-based methods are timeand effort-consuming and may raise bioethical issues, several in vitro methods have been proposed in order to identify stem-like properties of leukemic cells. Some of the most often used are: aldehyde dehydrogenase (ALDH) activity and Hoechst 33342 efflux assay [19] or divisional kinetics using PKH staining [20]. None of these methods seems currently superior in its precision to the limited-dilution transplantation, but each of them can serve as a rapid screening technique for new candidates for stem cells in a number of malignancies, not necessarily of haematological origin.

As the aforementioned in vitro functional assays rely on the natural properties of stem cells in general, they might useful for distinguishing between HSC and LSC only if combined with other methods, e.g. immunophenotyping. This is best seen in the case of ALDH activity, where normal haematopoietic stem cells within the bone marrow of AML patients have been shown to present usually a higher ALDH activity level than co-existing leukaemic stem cells [17].

\section{Genetic profile of leukaemia stem cells}

Following the introduction of microarray technology and subsequent modern next-generation sequencing (NGS) methods (reviewed in [21]), much effort has been devoted to identify genomic [22], transcriptomic [23, 24], and epigenetic [25] signatures of LSC (reviewed in [26]). The importance of these approaches has been underscored by the finding that stem cell gene expression programs have a profound effect on the clinical outcome in human leukaemia [8].

During sequencing of the $\mathrm{AML}$ genome, for instance, two distinct classes of somatic genetic alterations were recognised, among other variations. The first group is related mostly to the increased proliferative capabilities of LSC, and includes such genes as FLT3, Ras, or c-Kit. The second set of mutations contains alterations that induce maturation block, such as changes in the sequence of CEBPA, CBFB-MYH11, NPM1, DEK-NUP214, PML-RARA, MLL-MLLT3, or RUNX1-RUNX1T1 [27]. Additionally, deviations in the sequences of epigenetic modifiers (such as IDH1, IDH2, and DNMT3A) have been identified [28]. The image emerging from these experiments supports the 'two-hit' hypothesis of LSC formation. However, genetic studies also show that LSCs display a high degree of genetic variety [29], which reduces the chances for prospective development of a single universal anti-LSC treatment modality.

An interesting observation provided by next-generation techniques is that age-related changes in HSC, e.g. within the TET2 gene, contribute strongly to the pathogenesis of monoclonal B cell lymphocytosis (MBL) and chronic lymphocytic leukaemia (CLL) [30]. This raises hope for potential development of early screening methods capable of identifying patients with increased risk of development of mature lymphoid malignancy or perhaps the therapeutic prevention strategies against these disorders.

\section{Biological and molecular basis for 'stemness' in haematopoietic malignancies}

\section{LSC Niche}

Normal HSC residing in bone marrow (BM) depend heavily on support from specialised and strictly organised stem-cell niches, endosteal and vascular/peri-sinusoidal. The dialogue with other components of the niche is crucial for HSC self-renewal, survival, and functioning. Multiple extracellular and intracellular factors contribute to this phenomenon (reviewed in [31]), including haematopoietic growth factors (such as interleukin (IL)-3, IL-6, Flt3-ligand, LIF, TPO, SCF) and their cognate receptors, signalling pathways (e.g. Wnt- or Notch-mediated), molecules regulating cell cycle (e.g. p53), and numerous transcription factors or regulators (such as HOXB4, SCF/TEL-1, RUNX1, STAT5 [32]). Recently collected evidence suggests that LSCs robustly benefit from the support of bone marrow niches [33]. Leukaemia stem cells hijack the BM niche structures for the needs of their leukaemic growth and thus suppress the normal haematopoiesis. Thanks to their interactions with the endosteal niche, LSC can maintain their dormant state and are protected from the cytotoxicity of classical chemotherapy [34]. Leukaemia stem cells are retained in the BM niche primarily via interactions of CXCL12 chemokine (also known as stromal cell derived factor-1 - SDF1) with its cognate receptor CXCR4 [35], if it is expressed on LSC. These interactions are responsible for upregulation of the expression of adhesion molecules VCAM1 and VLA4. Some role has been also proposed for CD44, acting as an adhesion molecule and a receptor for osteopontin within the BM niche (reviewed in [36]).

\section{Self-renewal pathways}

As mentioned above, the aid provided by the microenvironment of the BM niche induces within LSC signals transduced via the intracellular pathways responsible for self-renewal of the stem cells (reviewed in [37]). These include phosphoinositide-3-kinase (PI3K)-, homeobox (HOX)-, Notch-, Hedgehog-, and Wnt/ $\beta$-catenin-mediated cellular signalling. Also important for LSC quiescence are the signals transduced via transforming growth factor $\beta$ (TGF- $\beta$ ) superfamily signalling and molecules of the SMAD family [37].

\section{Cell survival and proliferation pathways}

Aberrant, sustained activity of two major signalling pathways, nuclear factor (NF)-кB [38] and PI3K/Akt/mammalian target of rapamycin (mTOR) [39], in orchestration with a set of antiapoptotic molecules (e.g. Bcl-x and Bcl-2 [40]), are held responsible for the uncontrolled growth and decreased apoptosis in LSC.

\section{Therapeutic targeting of haematopoietic cancer stem cells}

Increasingly better phenotyping of LSC along with the growing understanding of the events leading to transformation from normal HSC to a malignant LSC, and then self-renewal and survival of LSC, have led to identification 
of a range of potential therapeutic targets in haematopoietic cancers [41].

Before going into more detail, it is crucial to understand two important aspects of therapeutic targeting against LSC. First, the above-mentioned signalling pathways create a dense, overlapping, and cross-talking network of molecular events within LSC. That translates into the fact that targeting a single event/pathway within LSC would, in many cases, result in only transient success. It is necessary to use the personalised medicine approach to clearly describe the aberrant networking within LSC of a given patient in order to tailor the best-fit set of targeted therapies in the particular case. Second, monitoring of the immediate outcome of anti-LSC therapies is far more complex than a standard classification into no response, partial response, or complete response etc., usually applied to describe the effectiveness of chemotherapy. It has become clear that assessment of anti-LSC effectiveness requires more sophisticated and precise methods [42].

Strategies for targeting LSC can be divided in two manners. The first division takes into consideration the chemical properties of the therapeutic agent. Here, two major classes emerge: small molecules (e.g. targeting the kinase activity of an oncogene or blocking molecular interactions) or antibody-/protein fusion-based drugs (targeting mostly surface molecules or extracellular growth factors). The second type of classification relates to the type of target. Here, we distinguish agents targeting adhesion molecules, growth factors and their receptors, intracellular signalling molecules, antiapoptotic molecules, or transcription factors.

Multiple targeted strategies have been attempted experimentally since the first identification of LSC. Only some of them have reached the clinical trial stage. The most prominent success has been achieved with BCR-ABL oncoprotein inhibitors in CML (although their effectiveness against LSC seems to be only partial [see below]), and many attempts are currently being undertaken to reproduce this success in other haematological malignancies. The examples applied in leukaemia treatment are presented in Table 1.
What must be remembered, again, is that even in the case of clearly identified oncogenic lesion, like BCR-ABL fusion protein in CML, the LSC can still develop overlapping pathways to ensure survival under unfavourable conditions, as is the case in CML. Recent data suggest that $C M L L S C S$ are relatively resistant to $B C R-A B L$ kinase-targeting drugs [43] and may require the addition of e.g. PI3K[44] or mTOR-targeted [45] strategies in order to achieve high level of LSC eradication. That underscores the need for systems biology-based analysis of each case and the combinatory, personalised approach to the targeted treatment of each patient suffering from haematological malignancy. It is also worth to mention the need for profound understanding of interactions of LSC with the patient's immune system in order to prospectively develop active immunisation strategies that can help eliminate minimal residual disease [46].

\section{Future perspectives}

When in 1971 Richard Nixon declared the "war on cancer", it was hoped that malignancies would be eradicated within the next 20/30 years. In 2014, cancer still remains a major cause of death and its frequency has dramatically increased in developed societies. However, researchers in this war have won many battles. The area of stem cell studies in haematological malignancies has become one of the most vivid and dynamic topics in current oncology. From what we have learned about cancer stem cells, great hope has emerged for the development of highly successful anticancer therapies that might eventually combat most cases of this deadly disease. Many challenges remain, with the need for undisputable identification and quantification of LSC in each type of malignancy and finding the right therapeutic combinations to minimise the chance of recurrence. Nevertheless, much evidence suggests that forty-three years after Richard Nixon's declaration, we are on the right track to eradicate haematological cancer via targeting its stem cells.

Table 1. Examples of potential anti-LSC targeted agents under investigation in leukaemia (based on [47])

\begin{tabular}{|c|c|c|}
\hline Type of target/therapy & Targeted structure/pathway & Therapeutic agent(s) \\
\hline \multirow[t]{5}{*}{ Cell surface markers } & CD33 & $\begin{array}{c}\text { Gemtuzumab ozogamicin } \\
\text { CART33 }\end{array}$ \\
\hline & CD44 & Monoclonal antibody \\
\hline & CD47 & Monoclonal antibody \\
\hline & CD96 & Monoclonal antibody \\
\hline & CD123 & $\begin{array}{l}\text { Diphtheria toxin-IL-3 fusion protein } \\
\text { Monoclonal antibody }\end{array}$ \\
\hline \multirow[t]{3}{*}{ Pathway-targeted therapies } & Hedgehog signalling pathway & Sonidegib \\
\hline & NF-кB signalling pathway & $\begin{array}{c}\text { Parthenolide } \\
\text { Dimethylaminoparthenolide } \\
\text { Bortezomib }\end{array}$ \\
\hline & c-Kit, Src tyrosine kinases & Dasatinib \\
\hline Anti-apoptotic molecules & $\mathrm{BCl} 2$ & $\begin{array}{c}\text { ABT-199 } \\
\text { Oblimersen }\end{array}$ \\
\hline
\end{tabular}


The authors declare no conflict of interest.

This work was supported by a grant from the European Commission $7^{\text {th }}$ Framework Programme: FP7-REGPOT-2012 CT2012-316254-BASTION.

\section{References}

1. Iyer KS, Saksena VN. A stochastic model for the growth of cells in cancer. Biometrics 1970; 26: 401-10.

2. Jackson EB, Brues AM. Studies on a Transplantable Embryoma of the Mouse. Cancer Res 1941; 1: 494-8.

3. Makino S. Further evidence favoring the concept of the stem cell in ascites tumors of rats. Ann N Y Acad Sci 1956; 63: 818-30.

4. Pandolfi A, Barreyro L, Steidl U. Concise review: preleukemic stem cells: molecular biology and clinical implications of the precursors to leukemia stem cells. Stem Cells Transl Med 2013; 2: 143-50.

5. Gentry SN, Jackson TL. A mathematical model of cancer stem cell driven tumor initiation: implications of niche size and loss of homeostatic regulatory mechanisms. PLoS One 2013; 8: e71128.

6. Lapidot T, Sirard C, Vormoor J, et al. A cell initiating human acute myeloid leukaemia after transplantation into SCID mice. Nature 1994; 367: 645-8

7. Bonnet D, Dick JE. Human acute myeloid leukemia is organized as a hierarchy that originates from a primitive hematopoietic cell. Nat Med 1997; 3: 730-7.

8. Eppert K, Takenaka K, Lechman ER, et al. Stem cell gene expres sion programs influence clinical outcome in human leukemia. Nat Med 2011; 17: 1086-93.

9. van Rhenen A, Feller N, Kelder A, et al. High stem cell frequency in acute myeloid leukemia at diagnosis predicts high minimal resid ual disease and poor survival. Clin Cancer Res 2005; 11: 6520-7.

10. Jordan CT. Unique molecular and cellular features of acute myelogenous leukemia stem cells. Leukemia 2002; 16: 559-62.

11. Hauswirth AW, Florian S, Printz D, et al. Expression of the target receptor CD33 in CD34+/CD38-/CD123+ AML stem cells. Eur J Clin Invest 2007; 37: 73-82.

12. Herrmann H, Sadovnik I, Cerny-Reiterer S, et al. Dipeptidylpeptidase IV (CD26) defines leukemic stem cells (LSC) in chronic myeloid leukemia. Blood 2014; 123: 3951-62

13. Cox CV, Diamanti P, Evely RS, Kearns PR, Blair A. Expression of CD133 on leukemia-initiating cells in childhood ALL. Blood 2009; 113: 3287-96.

14. Chan WI, Huntly BJ. Leukemia stem cells in acute myeloid leukemia. Semin Oncol 2008; 35: 326-35.

15. van Rhenen A, van Dongen GA, Kelder A, et al. The novel AML stem cell associated antigen CLL-1 aids in discrimination between normal and leukemic stem cells. Blood 2007; 110: 2659-66.

16. Majeti R, Chao MP, Alizadeh AA, Pang WW, Jaiswal S, Gibbs KD Jr, van Rooijen N, Weissman IL. CD47 is an adverse prognostic facto and therapeutic antibody target on human acute myeloid leukemia stem cells. Cell 2009; 138: 286-99.

17. Schuurhuis GJ, Meel MH, Wouters F, et al. Normal hematopoietic stem cells within the AML bone marrow have a distinct and higher ALDH activity level than co-existing leukemic stem cells. PLoS One 2013; 8: e78897.

18. Bonardi F, Fusetti F, Deelen P, van Goslinga D, Vellenga E, Schuringa JJ. A proteomics and transcriptomics approach to identify leukemic stem cell (LSC) markers. Mol Cell Proteomics 2013; 12: 626-37.

19. Pearce DJ, Bonnet D. The combined use of Hoechst efflux ability and aldehyde dehydrogenase activity to identify murine and hu man hematopoietic stem cells. Exp Hematol 2007; 35: 1437-46.

20. Hendrikx PJ, Martens CM, Hagenbeek A, Keij JF, Visser JW. Hom ing of fluorescently labeled murine hematopoietic stem cells. Exp Hematol 1996; 24: 129-40.

21. Braggio E, Egan JB, Fonseca R, Stewart AK. Lessons from next-generation sequencing analysis in hematological malignancies. Blood Cancer J 2013; 3: e127.
22. Mardis ER, Ding L, Dooling DJ, et al. Recurring mutations found by sequencing an acute myeloid leukemia genome. N Engl J Med 2009; 361: 1058-66.

23. Gentles AJ, Plevritis SK, Majeti R, Alizadeh AA. Association of a leukemic stem cell gene expression signature with clinical outcomes in acute myeloid leukemia. JAMA 2010; 304: 2706-15.

24. Krivtsov AV, Wang Y, Feng Z, Armstrong SA. Gene expression profiling of leukemia stem cells. Methods Mol Biol 2009; 538: 231-46.

25. Bartholdy B, Christopeit M, Will B, et al. HSC commitment-associated epigenetic signature is prognostic in acute myeloid leukemia. J Clin Invest 2014; 124: 1158-67.

26. Wiseman DH, Greystoke BF, Somervaille TC. The variety of leukemic stem cells in myeloid malignancy. Oncogene 2014; 33: 3091-8.

27. Naoe T, Kiyoi H. Gene mutations of acute myeloid leukemia in the genome era. Int J Hematol 2013; 97: 165-74.

28. Shih AH, Abdel-Wahab O, Patel JP, Levine RL. The role of mutations in epigenetic regulators in myeloid malignancies. Nat Rev Cancer 2012; 12: 599-612.

29. Wiseman DH, Greystoke BF, Somervaille TCP. The variety of leukemic stem cells in myeloid malignancy. Oncogene 2014; 33: 3091-8.

30. Kikushige Y, Miyamoto T. Hematopoietic stem cell aging and chronic lymphocytic leukemia pathogenesis. Int J Hematol 2014; 100: 335-40.

31. Morrison SJ, Scadden DT. The bone marrow niche for haematopoietic stem cells. Nature 2014; 505: 327-34.

32. Schepers H, Wierenga AT, Vellenga E, Schuringa JJ. STAT5-mediated self-renewal of normal hematopoietic and leukemic stem cells. JAKSTAT 2012; 1: 13-22.

33. Ishikawa F, Yoshida S, Saito Y, et al. Chemotherapy-resistant human AML stem cells home to and engraft within the bone-marrow endosteal region. Nat Biotechnol 2007; 25: 1315-21.

34. Zeng Z, Shi YX, Samudio IJ, et al. Targeting the leukemia microenvironment by CXCR4 inhibition overcomes resistance to kinase inhibitors and chemotherapy in AML. Blood 2009; 113: 6215-24.

35. Zhang Y, Patel S, Abdelouahab H, et al. CXCR4 inhibitors selectively eliminate CXCR4-expressing human acute myeloid leukemia cells in NOG mouse model. Cell Death Dis 2012; 3: e396.

36. Becker PS. Dependence of acute myeloid leukemia on adhesion within the bone marrow microenvironment. ScientificWorldJournal 2012; 2012: 856467.

37. Sands WA, Copland M, Wheadon H. Targeting self-renewal pathways in myeloid malignancies. Cell Commun Signal 2013; 11: 33.

38. Guzman ML, Neering SJ, Upchurch D, Grimes B, Howard DS, Rizzieri DA, Luger SM, Jordan CT. Nuclear factor-kappaB is constitutively activated in primitive human acute myelogenous leukemia cells. Blood 2001; 98: 2301-7.

39. Martelli AM, Evangelisti C, Chiarini F, Grimaldi C, McCubrey JA. The emerging role of the phosphatidylinositol 3-kinase/ akt/mammalian target of rapamycin signaling network in cancer stem cell biology. Cancers (Basel) 2010; 2: 1576-96.

40. Lagadinou ED, Sach A, Callahan K, et al. BCL-2 inhibition targets oxidative phosphorylation and selectively eradicates quiescent human leukemia stem cells. Cell Stem Cell 2013; 12: 329-41.

41. Roboz GJ, Guzman M. Acute myeloid leukemia stem cells: seek and destroy. Expert Rev Hematol 2009; 2: 663-72.

42. Mustjoki S, Richter J, Barbany G, et al. Impact of malignant stem cell burden on therapy outcome in newly diagnosed chronic myeloid leukemia patients. Leukemia 2013; 27: 1520-6.

43. Hamilton A, Helgason GV, Schemionek M, et al. Chronic myeloid leukemia stem cells are not dependent on Bcr-Abl kinase activity for their survival. Blood 2012; 119: 1501-10.

44. Airiau K, Mahon FX, Josselin M, Jeanneteau M, Belloc F. PI3K/ mTOR pathway inhibitors sensitize chronic myeloid leukemia stem cells to nilotinib and restore the response of progenitors to nilotinib in the presence of stem cell factor. Cell Death Dis 2013; 4: e827.

45. Stoklosa T, Glodkowska-Mrowka E, Hoser G, Kielak M, Seferynska I, Wlodarski P. Diverse mechanisms of mTOR activation in chronic and blastic phase of chronic myelogenous leukemia. Exp Hematol 2013; 41: 462-9.

46. Ruben JM, Visser LL, Bontkes HJ, Westers TM, Ossenkoppele GJ, de Gruijl TD, van de Loosdrecht AA. Targeting the acute myeloid 
leukemic stem cell compartment by enhancing tumor cell-based vaccines. Immunotherapy 2013; 5: 859-68.

47. Pollyea DA, Gutman JA, Gore L, Smith CA, Jordan CT. Targeting acute myeloid leukemia stem cells: a review and principles for the development of clinical trials. Haematologica 2014; 99: 1277-84.

\section{Address for correspondence}

\section{Radoslaw Zagozdzon}

Department of Immunology

Centre for Biostructure Research

Medical University of Warsaw

Banacha 1A, F Building

02-097 Warsaw, Poland

e-mail: radoslaw.zagozdzon@wum.edu.pl 